\title{
Biscoumarin derivative for designing the WLED display applications
}

Cite as: AIP Conference Proceedings 2115, 030206 (2019); https://doi.org/10.1063/1.5113045

Published Online: 12 July 2019

A. G. Pramod, Y. F. Nadaf, C. G. Renuka, and M. Sucheta

\section{ARTICLES YOU MAY BE INTERESTED IN}

Diameter and strain dependent structural, electronic and optical properties of gallium phosphide nanowires

AIP Conference Proceedings 2115, 030178 (2019); https://doi.org/10.1063/1.5113017

Non-linear optical properties of $\mathrm{BiFeO}_{3}$ nanoparticles

AIP Conference Proceedings 2115, 030191 (2019); https://doi.org/10.1063/1.5113030

Design and development of multi 1-dimensional position sensitive detectors housingshielding setup for small angle neutron scattering instrument at Dhruva

AIP Conference Proceedings 2115, 030214 (2019); https://doi.org/10.1063/1.5113053

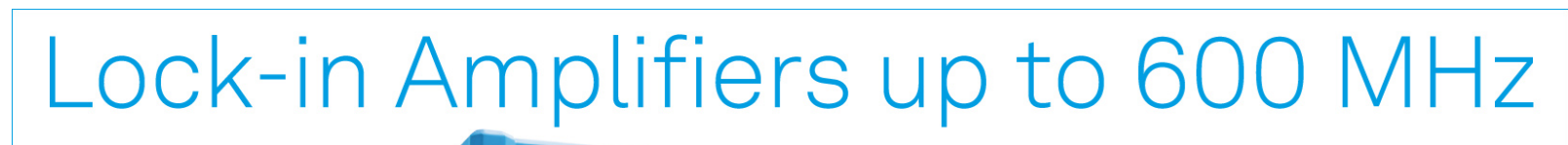

starting at

$\$ 6,210$

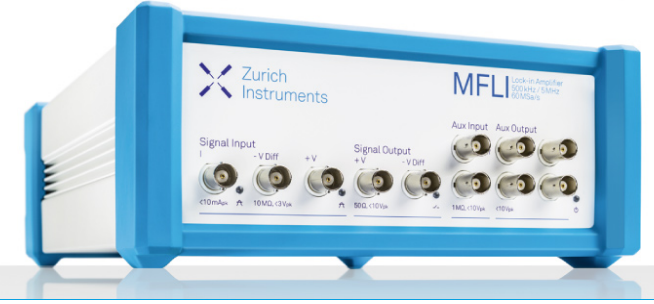

AIP Conference Proceedings 2115, 030206 (2019); https://doi.org/10.1063/1.5113045

(c) 2019 Author(s). 


\title{
Biscoumarin Derivative for Designing the WLED Display Applications
}

\author{
Pramod A G ${ }^{1}$, Nadaf $Y F^{2}$, Renuka $\mathrm{C} \mathrm{G}^{1 \mathrm{a})}$, Sucheta $\mathrm{M}^{2}$ \\ ${ }^{1}$ Department of Physics, Jnanabharathi Campus Bangalore University, Bengaluru-560056 \\ ${ }^{2}$ Department of Physics and Research Center, Maharani Science College for Women, Bengaluru-560001 \\ ${ }^{a)}$ Corresponding author: renubub@gmail.com
}

\begin{abstract}
The present technology for the demand of white light emission has received much importance due to their vast applications in various sensors, lightning devices and display etc. By mixing of green, blue and red, white light can produced. We synthesized 4-Hydroxy-3-[(4-hydroxy-2-oxo-2H-chromen-3-yl) (4-methoxyphenyl) methyl]-2Hchromen-2-one (4-HCMC) dye using acetic acid as a catalyst. In this present work we report, from the photoluminescence spectra duality nature of wavelengths in 4-HCMC molecule in different solvents is due to solvation effect, this results shows a simple extraction of dye in different solvents can be used to produce the desired wavelength. The 4-HCMC possesses high color purity, good CIE chromaticity coordinate, and they would have potential organic light emitting devices application, this simple method to produce the blue light as blue component can play important role in WLED.
\end{abstract}

\section{INTRODUCTION}

The coumarins and their derivatives were privileged oxygen heterocyclic compounds occurred in several natural products. Among various derivatives of coumarins, bis-coumarins have drawn much importance since they are well known to possess distinct pharmacological and biological properties viz., antifungal, anti-HIV, antibiotic, antiviral, antibacterial, anticoagulant, anticlotting and anticancer and also proved several applications in different fields [1]. Further, the novel biscoumarin derivatives has fascinated the scientific community due to the interesting applications of synthesized compounds (bis) such as red and green OLEDs have met the requirements for practical applications [2]. The literature revealed that, the different kinds of methods have been developed for the synthesis of biologically and spectroscopically active biscoumarin derivatives, specially the catalytic method. Therefore, we attempted to synthesis 4-Hydroxy-3-[(4-hydroxy-2-oxo-2H-chromen-3-yl) (4-methoxyphenyl) methyl]-2H-chromen-2-one (4-HCMC) dye using acetic acid as a catalyst [1-2]. Furthermore, the electronic spectra of 4-HCMC derivative in solutions depending on the solvents parameters they showed excellent fluorescence properties. These derivatives also exhibit the phenomena such as inter molecular charge transfer (ICT) and excited state intermolecular proton transfer which were played a major role in view of excellent photophysical properties like large stoke shifts, significant photostability and intense luminescence [4]. However, in literature there is no considerable report on the fluorescence studies of 4-HCMC bis coumarin compound. To this end, the present paper reports on the fluorescence properties, Commission Internationale de I'Eclairage (CIE) and color purity of previously mentioned compound dissolved in different solvents.

\section{EXPERIMENTAL}

\section{Materials and Methods}

The spectroscopic grade solvents such as propanol, 2Methoxyethanol (2ME), cyclohexane, carbon tetrachloride (CCL) and chloroform were purchased from the local chemical supplier having $99.9 \%$ purity were used without 
purification. The steady-state fluorescence experiment was carried out making use of Hitachi F-2700 Fluorescence Spectrofluorometer. The selected excitation wavelength is used for recording the fluorescence emission and obtained more resolution at the highest intensity. The experiment (all measurements) were carried out at 274 Kelvin with solution concentration of $10^{-6}-10^{-7} \mathrm{M}$ to overcome inner filter effect and self-aggregation effect.

\section{Synthesis Procedure}

We synthesized biscoumarin by catalyst method choosing the acetic acid as a catalyst. The mixture of aryl aldehydes (1mmol), 4-hydroxycoumarin $(3 \mathrm{mmol})$ and Acetic acid $(12 \mathrm{~mL})$ was taken in a $100 \mathrm{~mL}$ beaker and refluxed for 1-1.2h. By using thin layer chromatography (TLC) (Eluent: n-hexane and Ethyl acetate) the progress of the reaction was monitored. The vanishing of solvent in vacuum afforded the crude product which was recrystallized from ethanol to get 4-Hydroxy-3-[(4-hydroxy-2-oxo-2H-chromen-3-yl) (4-methoxyphenyl) methyl]$2 \mathrm{H}$-chromen-2-one (4-HCMC) biscoumarins. The optimized structure of synthesized 4-HCMC is presented in Fig. 1.

A white solid; 1H NMR (CDCl3 $400 \mathrm{MHz}): \delta 3.79$ (s, 3H, OCH3), $6.04(\mathrm{~s}, 1 \mathrm{H}, \mathrm{CH}), 6.88-7.69(\mathrm{~m}, 12 \mathrm{H}, \mathrm{Ar}-\mathrm{H})$, $8.00(\mathrm{~s}, 1 \mathrm{H}, \mathrm{OH}), 8.07(\mathrm{~s}, 1 \mathrm{H}, \mathrm{OH}) \mathrm{ppm} .13 \mathrm{C} \mathrm{NMR}(\mathrm{CDCl} 3100 \mathrm{MHz}): \delta 35.7,55.8,114.01,116.63,124.39$, $124.88,126.95,127.65,132.74,146.92,147.67,152.24,158.45,163.92,164.25$ ppm. HRMS: $[\mathrm{M}+\mathrm{H}]=\mathrm{m} / \mathrm{z}$ 443.1649; Anal. Calculated for C26 H18O7: C, 70.59; H, 4.11; found: C, 70.56; H, $4.09 \%$.

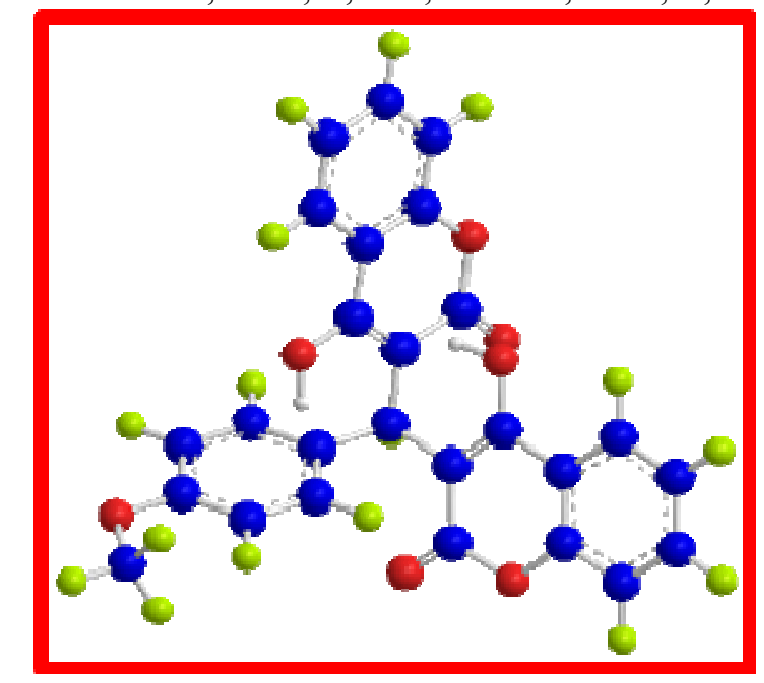

FIGURE 1. Optimized Structure of 4-HCMC Derivative.

\section{RESULTS AND DISCUSSION}

We have studied the photoluminescence (PL) emission spectra of the synthesized 4-HCMC coumarin derivative in different organic solvents of varying dielectric constant and refractive index such as propanol, 2ME, cyclohexane, CCL and chloroform. The PL spectra of synthesized 4-HCMC presented in Fig. 2. The sample was excited at 314 $\mathrm{nm}$ wavelength and the corresponding PL spectra shows the dual emission peaks observed in all the studied solvents except in cyclohexane it shows multiple emission peaks [3]. In case of propanol and 2ME solvents, the first peak is obtained at around $300 \mathrm{~nm}$ and the second peak which is high intense and sharp peak at around $340 \mathrm{~nm}$. In case of chloroform and carbon tetrachloride the emission peak again dual peak nature, the first peak is observed at $406 \mathrm{~nm}$ and the second peak is observed at $431 \mathrm{~nm}$. Whereas, in case of cyclohexane the emission peak observed consists of more than three peaks the first peak is observed at $340 \mathrm{~nm}$ (which is similar to propanol and 2ME's second peaks) the second peak is observed at $400 \mathrm{~nm}$ and the last peak was observed at $425 \mathrm{~nm}$ respectively (these are similar peaks which are obtained in case of chloroform and carbon tetrachloride solvents). Also from Fig 2, the emission spectra showed a slight bathochromic shift (red shift) in all studied solvents, these shifts are probably due to increase in the polarity of the solvents indicates $\pi \rightarrow \pi^{*}$ transitions are involved and also suggesting that the involvement of photo induced intermolecular charge transfer (ICT) state [4, 5], apart from solvent polarity, the hydrogen bonding effect also plays a major role for the shift of emission bands. And also the observed phenomenon i.e., duality nature 
of wavelengths in 4-HCMC molecule in different solvents is due to solvation effect, this results shows a simple extraction of dye in different solvents can be used to produce the desired wavelength [5].

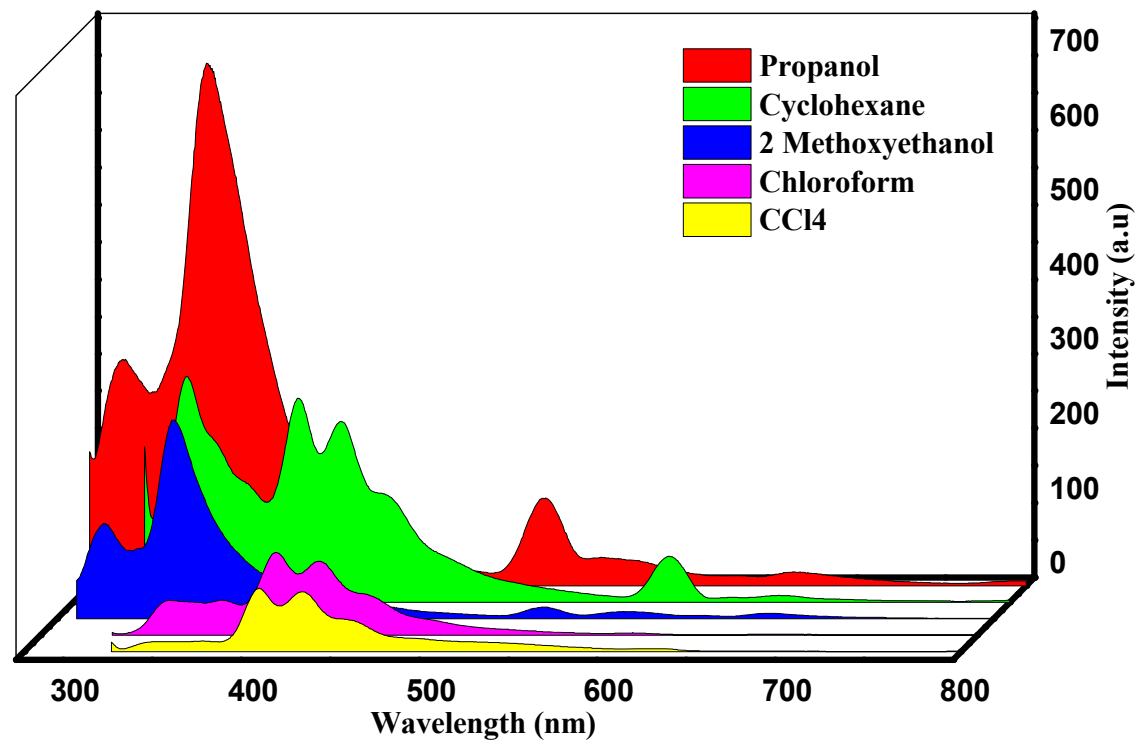

FIGURE 2. The photoluminescence emission spectra of 4-HCMC

The Commission Internationale de I'Eclairage (CIE) color coordinate values has been determined by using Osram Sylvania CIE co-ordinates software [6]. From the Fig 3 it is clear that the CIE coordinates lies in deep blue region for chloroform, cyclohexane, carbon tetrachloride and 2ME the corresponding $\mathrm{x}$ and $\mathrm{y}$ values are tabulated in Table 1. Whereas in propanol the CIE coordinate value is shifts towards yellowish green color. The color purity is an important property to estimate the possibility of the newly synthesized 4-HCMC derivative for OLEDs and display applications [7]. The color purity of the 4-HCMC is calculated using the following equation
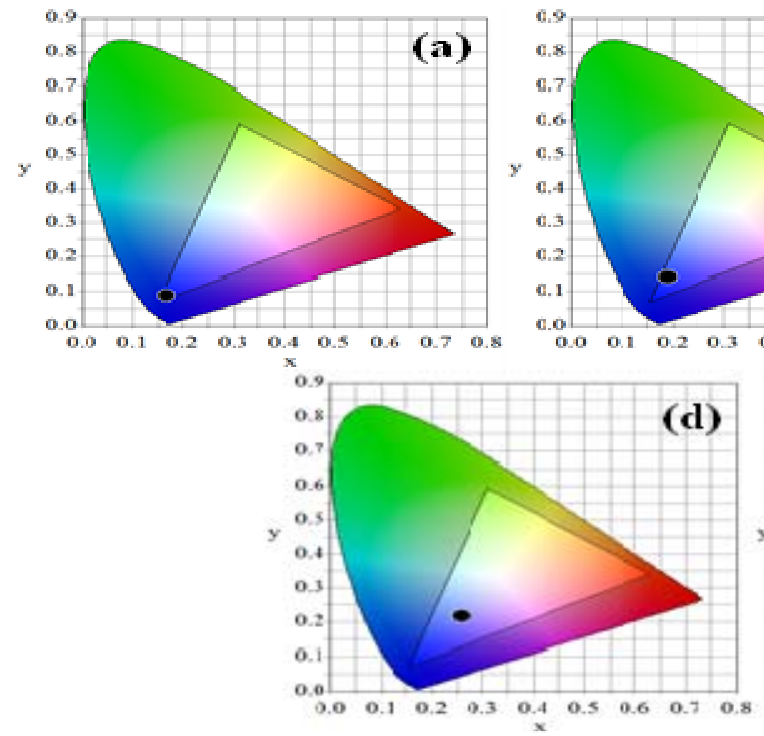

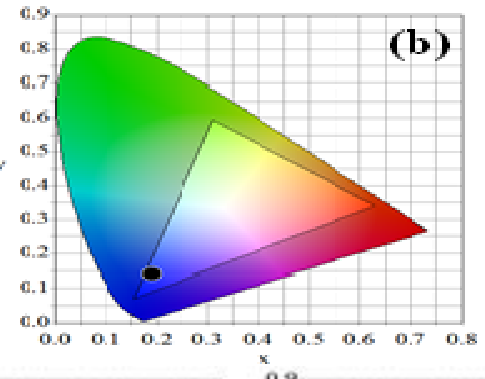

(b)
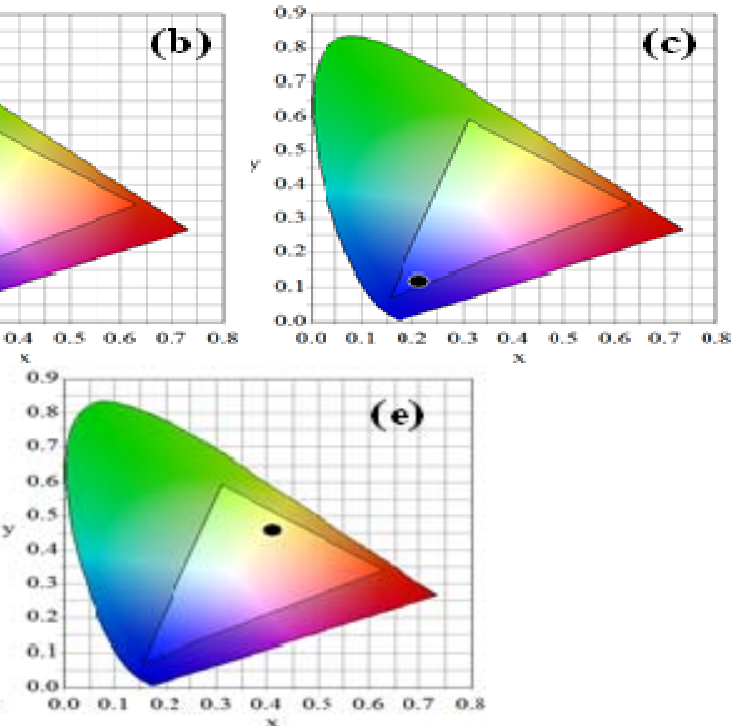

FIGURE 3. CIE Plots of 4-HCMC in (a) Chloroform, (b) CCl, (c) Cyclohexane, (d) $2 \mathrm{ME}$ and (e) Propanol Solvents.

$$
\text { Color purity }=\frac{\sqrt{\left(\mathrm{X}-\mathrm{X}_{\mathrm{i}}\right)^{2}+\left(\mathrm{Y}-\mathrm{Y}_{\mathrm{i}}\right)^{2}}}{\sqrt{\left(\mathrm{X}_{\mathrm{d}}-\mathrm{X}_{\mathrm{i}}\right)^{2}+\left(\mathrm{Y}_{\mathrm{d}}-\mathrm{Y}_{\mathrm{i}}\right)^{2}}} \times 100 \%
$$


Where, $(\mathrm{X}, \mathrm{Y})$ are CIE chromaticity coordinate values of studied compound, $\left(\mathrm{Xi}, \mathrm{Y}_{\mathrm{i}}\right)$ is the color coordinate of white illumination and $\left(\mathrm{X}_{\mathrm{d}}, \mathrm{Y}_{\mathrm{d}}\right)$ is the CIE coordinate of the dominant emission wavelength. In this report the $\left(\mathrm{X}_{\mathrm{i}}, \mathrm{Y}_{\mathrm{i}}\right)=$ $(0.310,0.316),\left(X_{d}, X_{d}\right)=(0.14,0.08)$ and the $(X, Y)$ values are presented in Table 1. From Table 1 it shows the color purity is $91.13 \%, 71.32 \%, 67.54 \%, 53.71 \%$ and $52.26 \%$ at excitation wavelength of $314 \mathrm{~nm}$ for studied solvents.

However, this results revealed that the observed the color coordinates of 4-HCMC slightly vary with different solvents due to decrease in intensity as well as the area of the photoluminescence emission. Thus, the 4-HCMC possesses high color purity and good CIE chromaticity coordinate also they would have potential application organic light emitting devices, this simple method to produce the blue light as blue component can play important role. The calculated chromaticity coordinates for 4-HCMC in chloroform are near to standard chromaticity (NTSC) for excellent blue color and thus are promising blue producers for WLED application [8].

\begin{tabular}{lccccc}
\hline Solvents & $\lambda_{\mathrm{a}}$ & $\begin{array}{c}\boldsymbol{\lambda}_{\mathrm{e}} \\
(\mathbf{n m})\end{array}$ & \multicolumn{2}{c}{$\mathbf{C I E}$} & \multicolumn{2}{c}{\begin{tabular}{c} 
Coordinates \\
\cline { 4 - 5 }
\end{tabular}} & & & $\mathbf{x}$ & $\mathbf{y}$ & $\begin{array}{c}\text { Color purity } \\
(\%)\end{array}$ \\
\hline Chloroform & 314.01 & 431.02 & 0.1673 & 0.0879 & 91.13 \\
CCL & 312.72 & 405.95 & 0.1896 & 0.1416 & 71.32 \\
Cyclohexane & 313.66 & 280.6 & 0.214 & 0.1198 & 67.54 \\
2ME & 308.43 & 340.67 & 0.2583 & 0.2158 & 53.71 \\
Propanol & 305.13 & 285.56 & 0.4121 & 0.4573 & 52.26 \\
\hline
\end{tabular}

Table 1. CIE color coordinates with color purity of 4-HCMC in studied solvents

\section{CONCLUSION}

In this present work we have reported the simpler method of synthesizing new bis coumarin derivative. The photoluminescence emission spectra showed a slight bathochromic shift (red shift) in the studied solvents, these red shifts are probably due to increase in the polarity of the solvents and suggesting that the involvement of photo induced intermolecular charge transfer (ICT) state. These duality nature of wavelengths in 4-HCMC molecule in different solvents is due to solvation effect, this results shows a simple extraction of dye in different solvents can be used to produce the desired wavelength. The 4-HCMC possesses high color purity, good CIE chromaticity coordinate and they would have potential application in organic light emitting devices, this simple method to produce the blue light as blue component can play important role. The calculated chromaticity coordinates for 4-HCMC in chloroform are near to standard chromaticity for excellent blue color and thus are promising blue producers for WLED application.

\section{REFERENCES}

1. J.R. Yerrabelly, V. Chakravarthula, H. Yerrabelly, P.R. Chitneni, V.R. Batchu, J.B. Nanubolu, and R.K. Krishnan, Tetrahedron Lett. 56, 2180 (2015).

2. A.R. Kiasat and L. Hemat-Alian, Res. Chem. Intermed. 41, 873 (2015).

3. C.G. Renuka, K. Shivashankar, P. Boregowda, S.S. Bellad, M. V Muregendrappa, and Y.F. Nadaf, J. Solution Chem. 46, 1535 (2017).

4. A.G. Al-Sehemi, M. Pannipara, and A. Kalam, Spectrochim. Acta Part A Mol. Biomol. Spectrosc. 171, 97 (2017).

5. $\quad$ C.G. Renuka, Y.F. Nadaf, G. Sriprakash, and S. Rajendra Prasad, J. Fluoresc. 28(3) 839 (2018).

6. See Osram Sylvania for CIE co-ordinates software, (2017), available at: https:/www.sylvania.com/enus/toolsand-resources/Pages/LED-ColorCalculator-Version-History.

7. M. Mubeen, A.D. Deshmukh, and S.J. Dhoble, AIP Conference Proceedings 1953, 080032 (2018).

8. Xiaoyong Huang, Heng Guo, Bin Li J. Alloys Compd. 720, 29 (2017).

9. Yan Huang, Zhi Yun Lu, Qiang Peng, Ru-Gang Xie, Ming-Gui Xie. J. Mat. Sci. 40, 601(2005).

10. Song Peng, Yihuan Zhao, Caixia Fu, Xuemei Pu, Liang Zhou, YanHuang, Zhiyun Lu. Chem. Eur.J. 24, 8056 (2018). 